Research Paper

\title{
Heat-killed Lactobacillus Reuteri GMNL-263 Prevents Epididymal Fat Accumulation and Cardiac Injury in High-Calorie Diet-Fed Rats
}

Po-Hsiang Liao' ${ }^{1}$, Wei-Wen Kuo², Dennis Jine-Yuan Hsieh ${ }^{3}$,Yu-Lan Yeh ${ }^{4,5}$, Cecilia-Hsuan Day ${ }^{6}$, Ya-Hui Chen ${ }^{7}$, Sheng-Huang Chang8, V. Vijaya Padma ${ }^{9}$, Yi-Hsing Chen $7,10^{*}$, Chih-Yang Huang 1, 11,12*凶

1. Graduate Institute of Basic Medical Science, China Medical University, Taichung, Taiwan

2. Department of Biological Science and Technology, China Medical University, Taichung, Taiwan

3. School of Medical Laboratory and Biotechnology, Chung Shan Medical University, Taichung, Taiwan

4. Department of pathology, Changhua Christian Hospital, Changhua, Taiwan

5. Jen-Teh Junior College of Medicine, Nursing and Management, Miaoli, Taiwan

6. Department of Nursing, Mei Ho University, Pingguang Road, Pingtung, Taiwan

7. Department of Research and Development, GenMont Biotech Incorporation, Tainan, Taiwan

8. Department of Health, Tsao-Tun Psychiatric Center, Executive Yuan, Nantou, Taiwan

9. Department of Biotechnology, Bharathiar University, Coimbatore, India

10. Institute of Biomedical Science, National Chung Hsing University, Taichung, Taiwan

11. Graduate Institute of Chinese Medical Science, China Medical University, Taichung, Taiwan

12. Department of Health and Nutrition Biotechnology, Asia University, Taichung, Taiwan

* These authors contributed equally to this paper.

$\square$ Corresponding author: Chih-Yang Huang Ph.D., Graduate Institute of Chinese Medical Science, China Medical University, Taichung, Taiwan, No. 91, Hsueh-Shih Road, Taichung, 404, Taiwan. Tel: +886-4-22053366 ext 3313. Fax: +886-4-22032295. E-mail address: cyhuang@mail.cmu.edu.tw

() Ivyspring International Publisher. Reproduction is permitted for personal, noncommercial use, provided that the article is in whole, unmodified, and properly cited. See http://ivyspring.com/terms for terms and conditions.

Received: 2016.03.21; Accepted: 2016.07.01; Published: 2016.07.05

\begin{abstract}
High-calorie diet-induced obesity leads to cardiomyocyte dysfunction and apoptosis. Impaired regulation of epididymal fat content in obese patients has been known to increase the risk of cardiac injury. In our study, a lactic acid bacteria, Lactobacillus reuteri GMNL-263, was evaluated for its potential to reduce body weight and body fat ratio and to prevent heart injury in rats with high-fat diet-induced obesity. Lactic acid bacteria supplementation restored the cardiac function and decreased the physiological changes in the heart of the obese rats. In addition, the Fas/Fas-associated protein pathway-induced caspase 3/e Poly polymerase mediated apoptosis in the cardiomyocytes of the obese rats was reversed in the Lr263-treated rats. These results reveal that fed with Lr-263 reduces body fat ratio, inhibits caspase 3-mediated apoptosis and restores cardiac function in obese rats through recovery of ejection fraction and fractional shortening. Our results indicated that the administration of Lr263 lactic acid bacteria can significantly down-regulate body fat and prevent cardiomyocyte injury in obese rats.
\end{abstract}

Key words: Functional food; High-calorie diet; Obesity; Cardiac dysfunction

\section{Introduction}

Obesity is a metabolic disease characterized by an excess accumulation of fat and the presence of some genetic defects such as TMEM18, SH2B1 and GNPDA2 had been shown that associated with obesity in clinical research [1]. Moreover, obesity also induces other complication disease, i.e., hyperlipidemia, inflammation, oxidative stress, myocardial apoptosis, lipid metabolic disorders and insulin resistance, and these pathological factors are associated with increased risks for the development of cardiovascular diseases (CVD) [2-6].

High-calorie intake often leads to obesity, insulin resistance, apoptosis, oxidative stress, hypertension and induced endoplasmic reticulum (ER) stress, 
which lead to the development of CVD [7-9]. The molecular mechanism behind obesity-driven tissue injury in mice and rats fed high-calorie diets involves caspase and poly (ADP-ribose) polymerase (PARP) activation, inducing apoptosis and leading to cardiac dysfunction [10]. Other mechanisms, including the up-regulated apoptotic response and mitochondrial dysfunction, decrease the survival rate of cardiomyocytes in genetically obese mice [11]. Additionally, some studies have suggested that apoptosis plays an important role in the pathogenesis of CVD [12-14].

In cardiomyocytes, first apoptosis signal (Fas)and mitochondria-dependent apoptotic pathways are the major pathways leading to myocyte apoptosis [15, 16]. In our previous study observed that cardiac apoptosis through Fas-dependent pathway activation in Zucker rats, a genetic animal model for obesity. Fas ligand levels induce caspase- 8 activation and promote cardiomyocyte apoptosis, indicating the Fas receptor-dependent apoptosis pathway in obesity-associated heart disease [17]. Other reports also note that the up-regulation of the cardiac mitochondria-dependent apoptosis pathway via BCL2-Associated Agonist Of Cell Death (Bad) dephosphorylation and cytochrome $\mathrm{C}$ release is accompanied by a decreased expression of B-cell lymphoma 2 (Bcl-2), which is an anti-apoptotic factor present in obese rats [18]. Moreover, both the Fas-dependent pathway and the mitochondria-dependent pathway trigger caspase-3 activation and cause PARP cleavage [19].

A previous study indicated that the insulin-like growth hormone (IGF-1) signaling pathway regulated cardiomyocyte survival responses through by IGF-1 receptor (IGF-1R), and low IGF-1 levels are highly correlated with heart failure and myocardial infarction [20]. IGF-1 activates phosphatidylinositol-3 kinase/Akt (PI3K/AKT) pathway, which leads to the prevention of myocyte apoptosis [21]. More importantly, activated PI3K promotes Akt phosphorylation, which increases Bcl-2 expression to down-regulate cardiomyocyte apoptotic activity [21]. In obese rats, reduced body fat accumulation, inflammation, oxidative stress and increased PI3K/Akt activation prevent cardiomyocyte remodeling and cardiac dysfunction [22].

Effects of probiotic on many metabolic-related diseases and immune-regulation have been reported extensively in several in vitro, in vivo research and clinical trials [23-29]. Previous studies indicated that supplemental probiotic decreased low-density lipoprotein (LDL), total cholesterol levels and reduced body weight and fatty liver [30]. In our previous study, we found that oral lactic acid bacteria reduced blood glucose and prevented heart injury in rats with streptozotocin-induced diabetes mellitus (DM)[31]. Moreover, lactic acid bacteria supplementation inhibited the pro-inflammatory and inflammatory cytokines secreted in type 1 DM animal model [32] and down-regulated insulin levels in a type $2 \mathrm{DM}$ animal model[33, 34]. However, the application of probiotic for the prevention of cardiomyocyte apoptosis induced by high calorie-induced obesity and CVD have not been extensively explored [33].

Previous studies indicated that Lactobacillus reuteri GMNL-263 (Lr263) had some biologic effect such as down-regulated blood lipids in a hyperlipidemia hamster model [35], prevented liver injury and improved insulin resistance in high fructose-fed rats [36] and provided renal protection in diabetic rats [37]. Although these reports showed the biological effect of Lr263, there is no enough evidence to indicate Lr263 exhibits cardio protection. In this study, we established high-calorie diet animal model to determine the effect of oral heat-killed Lr263 in heart protection.

\section{Materials and methods}

\section{Preparation of bacterial suspensions}

Lactobacillus reuteri GMNL-263

(Lr263), deposited under the Bioresource Collection and Research Center (BCRC) accession no. BCRC 910452, was provided by the GenMont Biotech, Inc. Lr263 was statically grown in Man Rogosa Sharpe (MRS; BD Difco, Franklin Lakes, NJ) broth at $37^{\circ} \mathrm{C}$ for $18-20 \mathrm{~h}$. The bacterial culture was harvested using centrifugation $(4000 \times \mathrm{g}, 15 \mathrm{~min})$, washed twice with PBS, autoclaved, and resuspended to a final concentration of either $4.13 \times 10^{8}$ or $2.07 \times 10^{9}$ cells $/ \mathrm{kg} /$ day for daily oral administration to rats during the 4-8 weeks experiment.

\section{Animal model}

The animal experiment protocol (NO. 101-263-B) was approved by Institutional Animal Care and Use Committee of China Medical University. Sprague-Dawley rats which were from BioLASCO Taiwan Company Limited and set up for four groups randomly ( $\mathrm{n}=5$ each). The control group rats administered with normal saline $(\mathrm{N})$, group II was high-calorie diet-fed rats (HC), and groups III and IV were high-calorie diet rats supplemented with the

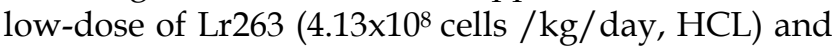
the high-dose of Lr263 (2.07×109 cells / kg/day, HCH). The dose of Lr263 was selected based on the recommended dietary allowance (RDA) for human (low-dose, 1 fold: $4 \times 10^{9}$ cells/day and high-dose, 5 folds: $2 \times 10^{10}$ cells / day) and calculated using the following equation: $4 \times 10^{9} / 60 \mathrm{~kg}$ (human weight) $\mathrm{X}$ 
$6.2($ rats conversion $)=4.13 \times 10^{8}, 5$ folds: $2.07 \times 10^{9}$ for rats. The animals were housed individually; the temperature was maintained at $20 \pm 2^{\circ} \mathrm{C} 1$ with $55 \pm 5 \%$ humidity. The rats were maintained on a 12-h dark-light cycle with lights on from 9 AM to 9 PM. The normal diet (AIN-76, protein $18.6 \%$, fat $10 \%$ and carbohydrates $60.6 \%$ ) was purchased from Young $\mathrm{Li}$ Company (Taipei, Taiwan); the high-calorie diet was the normal diet with added soybean oil $(8 \%)$ and condensed milk (44\%) to simulate the lifestyle diet. All rats had access to water ad libitum during the experimental period. Following the 8-week experimental period, all the rats were sacrificed.

\section{Echocardiography}

Rats were anesthetized by 3\% isoflurane and 97\% oxygen, fixed and supine on a acrylic board, shaved the chest hair. We measured the echocardiography by echocardiographic machine (ultrasound system -Vivid 7, GE Healthcare, Milwaukee, Wis). The heart imaged by the 2-dimensional mode near parasternal long-axis and short-axis view of the left ventricle obtained.Used these views were to measure the direct location of the M-mode cursor. M-mode echocardiographic examinations were conducted using a 6-15 $\mathrm{MHz}$ linear transducer (15-6 L) via a parasternal long-axis approach. LV M-mode measurements at the level of the papillary muscles included LV internal end-diastolic dimensions, LV internal end-systolic dimensions, interventricular septum and posterior wall thicknesses, ejection fraction (EF) and fractional shortening (FS).

\section{Physiological features measuring}

Rats were weighed every week during the experiment. The total body fat was measured from the surface of epididymal. The body fat ratio (\%) calculated using the following equation:

Body fat ratio $=($ total body fat $(\mathrm{g}) /$ body weight $(\mathrm{g})) \mathrm{X}$ 100

The food intake was weighed every day during the experiment. The food utilization rate (\%) was calculated using the following equation:

Food utilization rate $=($ total body weight gain (g)/total food intake (g)) X 100

\section{Hematoxylin and eosin staining}

The rat hearts tissue for each groups were incubated with formalin, next dehydrated in graded alcohol (100, 95 and 75\%) and embedded in paraffin wax. Cut tissue sample blocks into $0.2 \mu \mathrm{m}$-thick sections. Before staining incubated the tissue sections with xylene to deparaffinised. After deparaffinised the tissue sections were stained by hematoxylin and eosin and washed with water. The data collected using a Zeiss Axiophot microscope (OLYMPUS Microscopy).

\section{Tissue protein extraction}

The cardiac tissue extracts (left ventricular) of the rats in a lysis buffer $(1 \mathrm{mg} / \mathrm{ml})$. The sample buffers were placed on ice for $30 \mathrm{~min}$ and next centrifuged at $13500 \mathrm{~g}$ for $20 \mathrm{~min}$. The supernatants were collected and stored at $-80^{\circ} \mathrm{C}$ for use in further experiments. The composition of lysis buffer is $20 \mathrm{mM}$ Tris, $2 \mathrm{mM}$ EDTA, 50mM 2-mercaptoethanol, 10\% glycerol, protease inhibitor per $/ 10 \mathrm{ml}$ and phosphatase inhibitor $1 \mu \mathrm{l} / \mathrm{ml}$.

\section{Western blot analysis}

The protein sample buffer of the cardiac tissue extracts were determined by Lowry's protein assay method and load $15 \mu \mathrm{g}$ per sample in each well. To analysis protein samples by SDS-PAGE $(12 \%, 10 \%$ and $8 \%$ ) and supply of $100 \mathrm{~V}$. Next we transferred the protein onto the polyvinylidene difluoride (EMD Millipore Life Sciences) membranes by $90 \mathrm{~V}, 70 \mathrm{~min}$. The membranes were blocking by $5 \%$ fat-free milk in Tris-buffered saline (TBS) for 1 hour, after washed by TBS buffer the membranes incubated with primary antibodies to specific proteins : p-Akt (sc-5298, Santa Cruz Biotechnology), Bcl-2 (sc-7382, Santa Cruz Biotechnology), cleaved caspase 3 ( \#9664, Cell signaling), GAPDH (sc-25778, Santa Cruz Biotechnology) and dilution 1000x. After incubated with secondary antibodies the membranes were used for detection by with Fujifilm LAS-4000 (GE Healthcare Life Science).

\section{TUNEL assay}

The terminal deoxynucleotidyltransferase 2'-deoxyuridine 5'-triphosphate (dUTP)-mediated nick-end labeling (TUNEL) assay, tissue sections were dehydrated using graded alcohol $(70 \%, 90 \%, 100 \%, 10$ min for each), rinsed three times in xylene, and incubated with proteinase $\mathrm{K}$ for 30 min; washed with PBS, incubated with a permeabilization solution $(5 \%$ Triton buffer) and incubated in a blocking buffer for 1 hr; and finally washed twice with PBS. The sections were then incubated for $60 \mathrm{~min}$ at $37^{\circ} \mathrm{C}$ with the TUNEL assay buffer (Roche Applied Science). The TUNEL-positive nuclei were showed by green color detected by florescence microscope (OLYMPUS).

\section{Statistical analysis}

The results are reported as the means and standard deviations of three independent analysis experiments, for western blot analysis, the experiments were repeated three times with 
corresponding blots from 4 different tissue slices. Statistical analysis was performed using one-way ANOVA. ${ }^{*} \mathrm{P}<0.05,{ }^{* *} \mathrm{P}<0.01$ or ${ }^{* * *} \mathrm{P}<0.001$ : the mean values were significantly different from those of the $\mathrm{N}$ group, $\# \mathrm{P}<0.05, \# \# \mathrm{P}<0.01$ or \#\#\#P<0.001: the mean values were significantly different from those of the HC group.

\section{Results}

\section{Physiological features}

As shown in Table 1, the average body weight in the HC group was $573.4 \mathrm{~g}$ (sd 39.4), (19.4\% higher than in the $\mathrm{N}$ group); the food intake was $1226.6 \mathrm{~g}$ (sd $100.8)$, (15\% lower than in the $\mathrm{N}$ group); the food utilization rate was $14.6 \%$ (sd 3.2), (1.8-fold higher than that $\mathrm{N}$ group); and the epididymal fat content was $2.1 \%$ (sd 0.4), (1.75-fold higher than in the $\mathrm{N}$ group). The body weight of rats treated with low-dose Lr263 was $493.8 \mathrm{~g}$ (sd 32.1), (13.8\% lower than in the HC group); the food intake was $1189.8 \mathrm{~g}$ (sd 50.6), (2\% lower than in the HC group); the food utilization rate was $12.1 \%$ (sd 4.4), (0.8-fold lower than in the HC group); and the epididymal fat content was $1.8 \%$ (sd $0.4)$, (0.8-fold lower than in the HC group). Moreover, The body weight of the rats treated with high-dose Lr263 was $470.8 \mathrm{~g}$ (sd 27.9), (17.9\% lower than that HC group); the food intake was $1164.8 \mathrm{~g}$ (sd 29.5), (5\% lower than in the HC group); the food utilization rate was $12.6 \%$ (sd 4.4$)$, (0.86-fold lower than in the HC group); and the epididymal fat content was $1.4 \%$ (sd $0.4)$, (0.66-fold lower than in the HC group). These results indicated that Lr263 functional food treatment may prevent the detrimental effects of a high-calorie diet on the physiological features rats.

\section{Cardiac echocardiography}

Previous studies obtained that ejection fraction (EF (Teich)) and fractional shortening (FS) decreased in different heart disease models especially in heart failure [38-41], therefore, EF (Teich) and (FS) were considered as heart function markers.

After one month of Lr263 supplementation, the heart function markers: EF (Teich) and FS was determined by cardiac echocardiography. The average $\mathrm{EF}$ (Teich) in the control group rats was $80.0 \%$ (SD 2.5), and the average FS was $43.9 \%$ (SD 2.4) (Table 2). The average EF (Teich) of the high-calorie group was $71.9 \%$ (SD 5.4), (8\% lower than that of the $\mathrm{N}$ group rats), and the average FS was 38.7\% (SD 4.6), (5.2\% lower than that of the $\mathrm{N}$ group rats). The average EF (Teich) of the HCL group rats was $77.2 \%$ (SD 5), (5.2\% higher than that of the HC rats), and the average FS was $41.4 \%$ (SD 4.7), (2.7\% higher than that of the $\mathrm{HC}$ rats). The average EF (Teich) of the $\mathrm{HCH}$ rats was $80 \%$ (SD 2.5), (8.1\% higher than that of the
$\mathrm{HC}$ rats), and the average FS was $43.8 \%$ (SD 2.6), (5.1\% higher than that of the $\mathrm{HC}$ rats).

Table 1. Effects of body weight, food intake and food utilization rate and epididymal fat content on rats fed with a high-calorie diet and different cell counts of Lr263

\begin{tabular}{|c|c|c|c|c|}
\hline & $\mathrm{N}$ & $\mathrm{HC}$ & $\mathrm{HCL}$ & $\mathrm{HCH}$ \\
\hline Body weight (g) & $480.0 \pm 12.6$ & $573 . \pm 39.4^{*}$ & $493.8 \pm 32.1 \#$ & $470.2 \pm 27.9 \#$ \\
\hline $\begin{array}{l}\text { Epididymal fat } \\
\text { content }(\%)\end{array}$ & $1.2 \pm 0.1$ & $2.1 \pm 0.4^{*}$ & $1.8 \pm 0.4$ & $1.4 \pm 0.3 \#$ \\
\hline $\begin{array}{l}\text { Food utilization } \\
\text { rate }(\%)\end{array}$ & $7.7 \pm 1.1$ & $14.6 \pm 3.2^{*}$ & $12.1 \pm 4.4$ & $12.6 \pm 1.5$ \\
\hline Food intake (g) & $1444.2 \pm 61.6$ & $1226.6 \pm 100.8^{*}$ & $1189.8 \pm 50.6^{*}$ & $1164.4 \pm 29.5^{*}$ \\
\hline \multicolumn{5}{|c|}{ Data are expressed as the mean \pm standard deviation. } \\
\hline \multicolumn{5}{|c|}{ Mean values were significantly different from those of the $\mathrm{N}$ group: ${ }^{*} \mathrm{P}<0.05 . \mathrm{n}=5$} \\
\hline \multicolumn{5}{|c|}{ Mean values were significantly different from those of the HC group: $\# \mathrm{P}<0.05 . n=5$} \\
\hline \multicolumn{5}{|c|}{$\begin{array}{l}\text { N, normal control; HC, high-fat diet; HCL, high-fat diet and low dose of Lr263; } \\
\mathrm{HCH} \text {, high-fat diet and high dose of Lr263. }\end{array}$} \\
\hline
\end{tabular}

Table 2. Determine of cardiac physiological functions by echocardiography

\begin{tabular}{lllll}
\hline & EF\% (Teich) & & \%FS \\
\hline & Mean & SD & Mean & SD \\
$\mathrm{N}$ & 80.02 & 2.5 & 43.96 & 2.49 \\
$\mathrm{HC}$ & $71.99^{* *}$ & 5.42 & $38.72^{*}$ & 4.64 \\
$\mathrm{HCL}$ & $77.22 \#$ & 5.01 & 41.48 & 4.7 \\
$\mathrm{HCH}$ & $80.09 \# \#$ & 2.55 & $43.85 \#$ & 2.69 \\
\hline
\end{tabular}

Data are expressed as the mean \pm standard deviation.

Mean values were significantly different from those of the $N$ group: ${ }^{*}<<0.05 . n=5$ Mean values were significantly different from those of the $\mathrm{HC}$ group: \# $\mathrm{P}<0.05$, $\# \# \mathrm{P}<0.01 \mathrm{n}=5$

N, normal control; HC, high-fat diet; HCL, high-fat diet and low dose of Lr263; $\mathrm{HCH}$, high-fat diet and high dose of Lr263.

\section{Cardiac tissue biopsy}

The cardiac tissue sections of the high-calorie-induced obese rats stained with hematoxylin and eosin showed that the arrangement of the cardiomyocytes was disordered compared with the $\mathrm{N}$ group. After treatment with Lr263, the arrangement of the cardiomyocytes was more ordered than in the HC rats; the arrangement of the cardiomyocytes of the $\mathrm{HCH}$ rats was similar to that of the $\mathrm{N}$ group rats (Fig. 1).

\section{Protein marker analysis}

The important apoptosis and survival protein marker expression levels of the hearts were determined by Western blot analysis of the cardiac tissue extracts. In our results, the expression levels of the apoptosis marker caspase 3 and the downstream protein PARP in the hearts of the HC group rats were much higher than in the $\mathrm{N}$ group rats (Fig. 2A). The expression levels of the survival protein marker p-Akt and the downstream protein Bcl-2 were also down-regulated in the hearts of the $\mathrm{HC}$ group rats (Fig. 2A). The expression levels of caspase 3 and PARP of the HCL and $\mathrm{HCH}$ groups were lower than the hearts of the HC group rats. The expression levels 
of p-Akt and Bcl-2 in the hearts of the HCL and HCH groups were increased compared with the HC group and were even higher than the hearts of the $\mathrm{N}$ group rats. Moreover, analysis of the protein expression ratios indicated that, in the rat hearts, treatment with Lr263 prevented heart injury by the up-regulation of Bcl-2 and the down-regulation of caspase 3 and PARP proteins in a dose-dependent manner (Fig 2. C-E).

\section{Cardiac apoptosis assay}

The TUNEL-positive nuclei showed a higher number (6-fold higher than in the $\mathrm{N}$ group) of apoptosis in the heart tissue sections of the HC group rats (Fig 3. B). After treatment with low-dose and high-dose Lr263 showed that Lr263 down-regulated cardiomyocyte apoptosis. Moreover, Lr263 reduced the cardiomyocyte apoptosis in the $\mathrm{HCL}$ and $\mathrm{HCH}$ groups, with 3-fold and 4-fold lower apoptotic rates than in the HC group, respectively. Our results indicate that Lr263 prevents cardiomyocyte apoptosis induced by a high-calorie diet in a dose-dependent manner.

\section{Discussion}

Several studies showed lactic acid bacteria biological functions involved anti-inflammatory, antioxidant, anti-cancer, blood pressure and plasma lipid regulation activities [42-44]. Furthermore, lactic acid bacteria are known to down-regulate blood glucose levels and prevent complications of diabetes [45]. Other studies have shown that lactobacilli provide cardioprotection against ischemia and diabetes-induced apoptosis $[46,47]$. The high-calorie and high-fat diet-induced obesity animal model

(A)
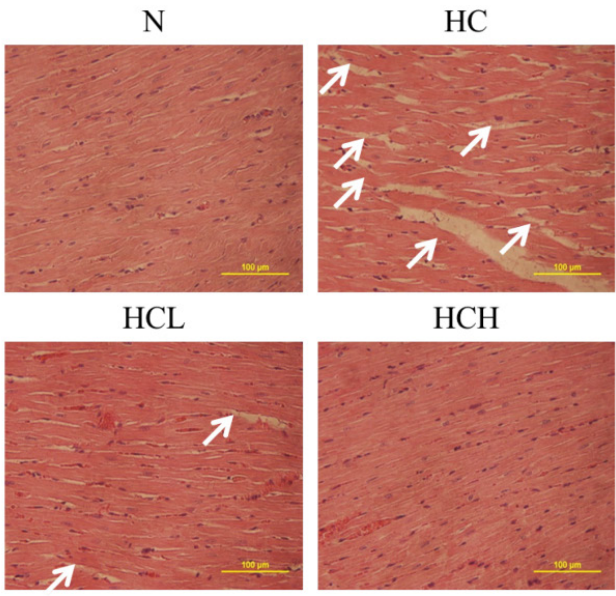

(B)
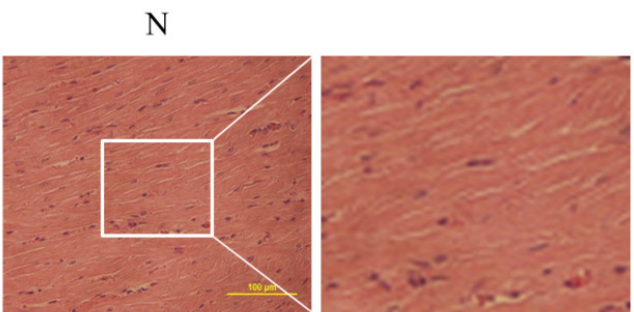

HCL
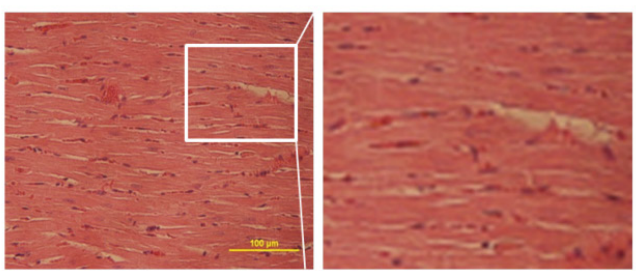

HC
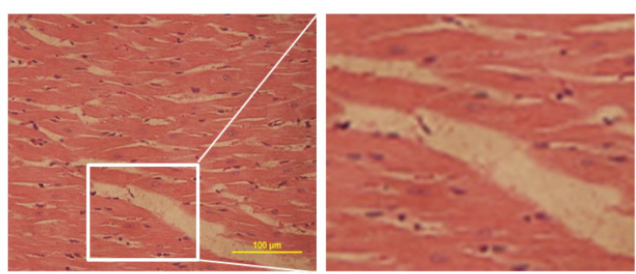

$\mathrm{HCH}$
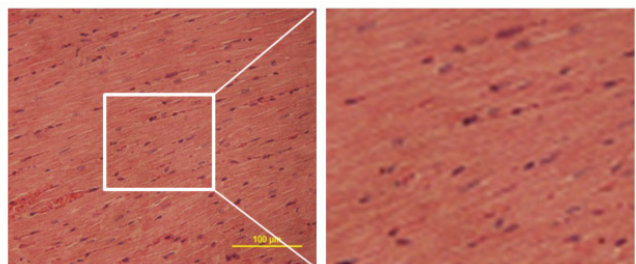

Figure 1 Hematoxylin and eosin staining of cardiac tissue sections. Histopathological analysis of cardiac tissue sections of the left ventricles of the control rats $(\mathrm{N})$, high-calorie-induced obesity rats without treatment (sham) $(\mathrm{HC})$, high-calorie-induced obesity rats treated with a low dose of $\mathrm{Lr} 263(\mathrm{HCL})$ and high-calorie-induced obesity rats treated with a high dose of Lr263 $(\mathrm{HCH})$. Cellular disorder area (White arrows). 
exhibits features of the pathophysiological disease as it occurs in human obesity, including diabetes, cardiac hypertrophy and cardiovascular dysfunction [48, 49]. Probiotics change the intestinal microflora profile and thereby brings change in the general metabolism. It is worthy to mention that we cannot predict the lactic acid bacteria growth number in rats intestinal, further other reports indicated both the live as well as dead lactic acid bacteria have potential beneficial effects $[50,51]$. So in this study we used the heat shock lactic acid bacteria to investigate the dose-specific effects of Lr263 in high calorie-fed rat hearts. Moreover, recent research has clearly indicated that feeding mice a high-fat diet was associated with a body mass increase and significant modifications in blood lipids and glucose homeostasis, fat accumulation and adipocyte remodeling [52]. However, the use of functional foods in the management of lifestyle diseases, particularly in obesity and CVD, has not been investigated extensively. In a healthy rat, the numerical value of EF is near 80 , and the FS value is higher than 40; however, in a healthy human, the numerical value of EF is near 55, and the FS value is higher than 30. EF or FS values below the normal range may indicate the development of cardiac dysfunction [53]. In this study, decreases in the values of EF (by $8.1 \%$ ) and in those of FS (by 5.1\%) reveal the onset of cardiac dysfunction. The cardiac dysfunction was also accompanied by deterioration in cardiomyocyte characteristics such as the cardiomyocyte derangement in rats with high-calorie-induced obesity.

(A)

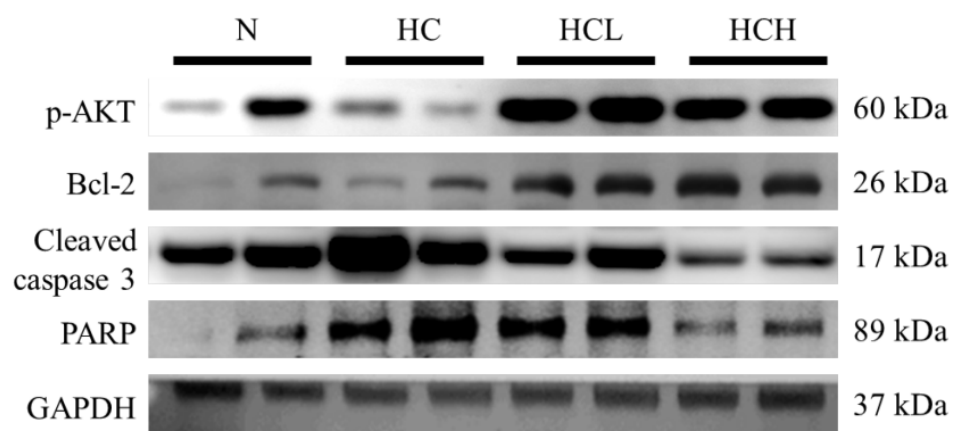

(B)

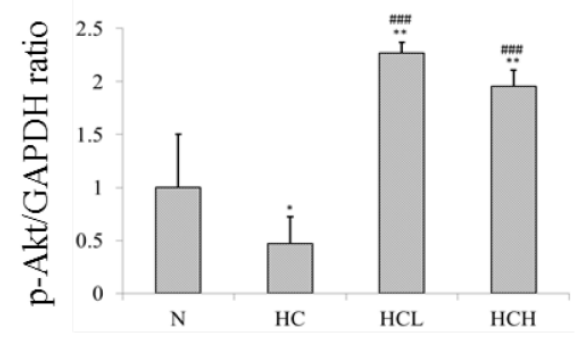

(D)

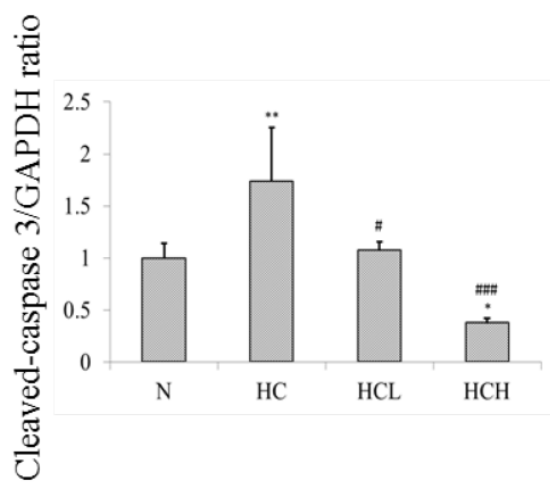

(C)

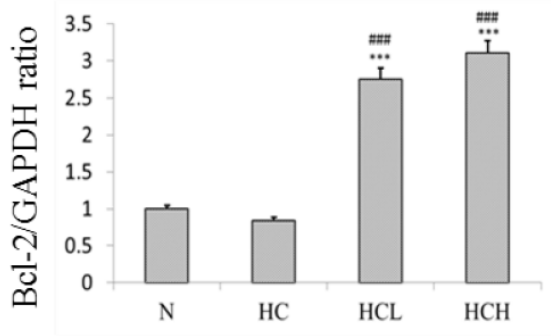

(E)

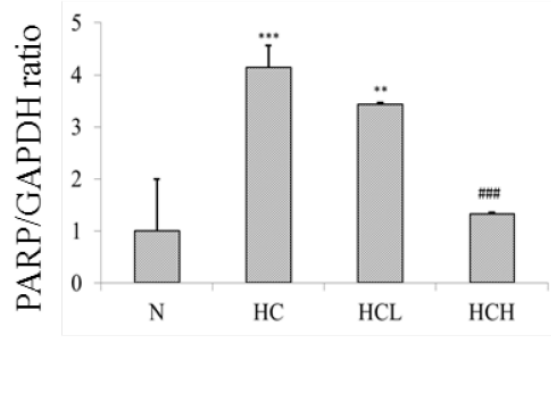

Figure 2 Protein expression analysis by Western blotting. The level of apoptosis marker proteins increased and survival marker proteins decreased in the left ventricles of the rats with high-calorie-induced obesity, whereas treatment with low dose and high dose of Lr263 reduced the expression of apoptotic proteins and increased survival proteins. (A) Western blots of caspase 3/PARP and $\mathrm{p}-\mathrm{Akt} / \mathrm{Bcl} 2$ proteins. (B-E) The densitometry of the Western blots shown in (A). Quantitative analysis of the expression levels of (B) p-Akt, (C) $\mathrm{Bcl} 2$, (D) caspase 3 and (E) PARP was performed by normalization using GAPDH as the internal control. Data represent the results of four animal models; the samples from two rats were pooled together, and therefore, four independent experiments were conducted. Mean values were significantly different from those of the $N$ group: $* P<0.05, \quad * * P<0.01$ and $* * * \mathrm{P}<0.001$. Mean values were significantly different from those of the HC group: \#P<0.05, \#\#P<0.01 and \#\#\#<0.001. 


\section{(A)}
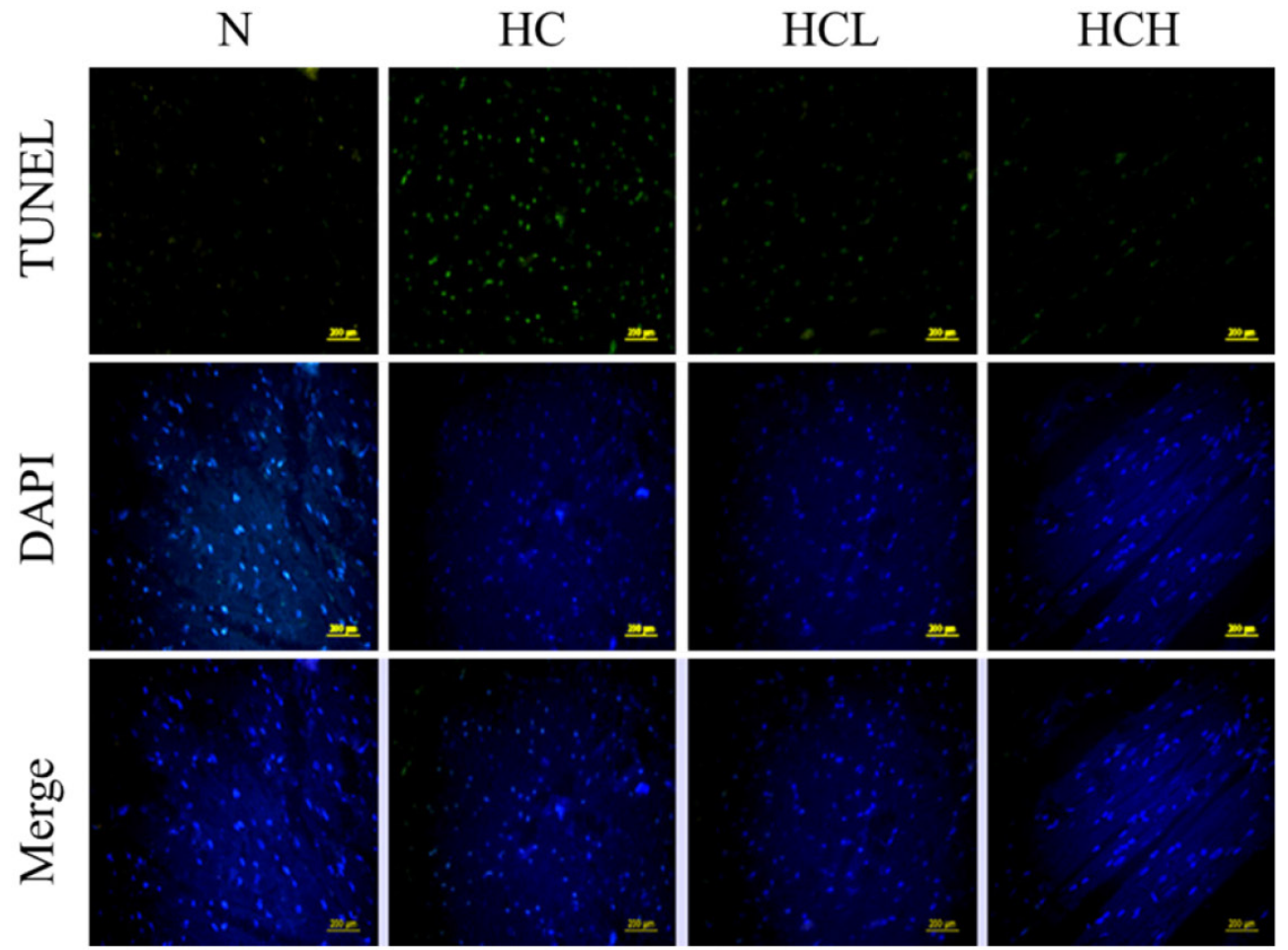

(B)

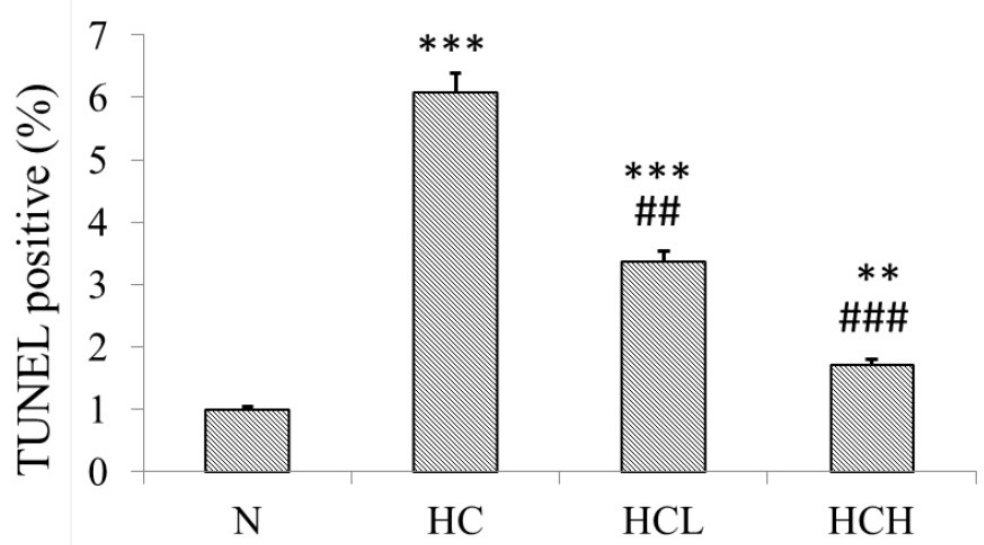

Figure 3 TUNEL and DAPI staining to detect cardioapoptosis. (A) DAPI- and TUNEL-stained heart tissue sections of the control rats, high-calorie induced-obesity rats and rats treated with low and high doses of Lr263. The nuclei were stained blue by DAPI staining, and the DNA fragments were stained green by the TUNEL assay. (B) Quantitative analysis of the percentage of apoptosis as detected by the TUNEL assay. Mean values were significantly different from those of the $\mathrm{N}$ group: $* \mathrm{P}<0.05, * * \mathrm{P}<0.01$ and $* * * \mathrm{P}<0.001$. Mean values were significantly different from those of the $\mathrm{HC}$ group: \#P<0.05, \#P<0.01 and \#\#P<0.001.

However, treatment with a high dose of Lr263 (2 $x 10^{10}$ cell counts) maintained the percentage of epididymal fat at $1.4 \%$ and controlled the body weight at $470.2 \mathrm{~g}$ in the rats with high-calorie-induced obesity. Moreover, the EF and FS values were above $80 \%$ and $43 \%$, similar to the control group (Table 2). Furthermore, the hematoxylin and eosin staining analysis of the biopsies showed that treatment with Lr263 decreased cardiac injury, and the hearts of the rats in the high-dose group were similar to the control group. Therefore, supplementation with Lr263 is found was found to provide cardioprotection in obese rats.

In control group epididymal fat content in healthy rats is approximately $1 \%$ of the body weight, the average epididymal fat content in the rats with high-calorie induced obesity was observed to be above 2.1\%, and Lr263 treatment reduced the epididymal fat content to $1.8 \%$ in the low-dose group and $1.4 \%$ in the high-dose group. Although 
epididymal fat content was not down-regulated to normal levels after Lr263 treatment in all rats, the adverse symptoms of the obesity group were reduced. Thus, high-calorie induced obesity may only increase risk when the epididymal fat content rises above $2.1 \%$, and therefore, control of the epididymal fat content is a key target to evaluate fat accumulation in obese patients.

Obesity is a strong risk factor for the development of cardiac dysfunction, particularly hypertrophy. Obesity has been shown to up-regulate BNP protein, a key factor in the development of hypertrophy, which may result in heart failure[54]. The characteristics of heart failure involve progressive left ventricular dysfunction and loss of cardiomyocytes via necrosis or apoptosis [55]. Previous studies have shown that obesity increases the incidence of heart disease by $50 \%$ compared with humans at their target weight [56]. In our recent studies, the major pathways leading to the activation of the cleavage of caspase 3 and PARP, resulting in cardiac dysfunction $[47,57,58]$.

Caspase 3 and PARP cleavage and activation were significantly suppressed in a dose-dependent manner by low-dose $\left(4.13 \times 10^{8}\right.$ cells $/ \mathrm{kg} /$ day $)$ and high-dose $\left(2.07 \times 10^{9}\right.$ cells $/ \mathrm{kg} /$ day) Lr263 treatments (Fig. 2). Moreover, the expression levels of p-Akt and Bcl-2 were also significantly up-regulated by Lr263 treatment (Fig. 2), although biopsy analysis of the heart damage in rats with high-calorie induced obesity showed a dose-dependent reversal after Lr263 treatment (Fig. 1). In addition, the TUNEL assay showed that Lr263 supplementation reduced the percentage of apoptosis in rat hearts induced by a high-calorie diet. These results indicated that Lr263 treatment decreased the body fat ratio and down-regulated the expression of caspase 3 and PARP, preventing cardiac dysfunction in obese rats. The mechanism of Lr263-mediated cardioprotection and body fat control in obese rats requires further investigation.

Our experimental results clearly indicate that high-calorie diets successfully induce obesity in rat models, leading to cardiac dysfunction and body fat accumulation. Importantly, supplementation with Lr263 reduced body fat accumulation and body weight. Moreover, our data show that treatment with Lr263 prevented cardiac injury in obesity animal models. This finding indicates that Lr263 could be used as a complementary therapy in the prevention and treatment of cardiac disorders and the possible mechanisms may via decreased apoptosis protein (cleaved caspase 3 and PARP) expression and up-regulated survival protein (p-Akt and Bcl-2) expression and cardiac function (EF\% and $\% \mathrm{FS})$.

\section{Acknowledgments}

This study was supported by the Taiwan Ministry of Health and Welfare Clinical Trial and Research Center of Excellence (MOHW105-TDU-B212-133019).

\section{Competing Interests}

The authors have declared that no competing interest exists.

\section{References}

1. Chen PY, Lu ML, Huang MC, Kao CF, Kuo PH, Chiu CC, et al. The Relationships of Obesity-Related Genetic Variants With Metabolic Profiles and Response to Metformin in Clozapine-Treated Patients With Schizophrenia. Journal of clinical psychopharmacology. 2015; 35: 574-8.

2. Hedley AA, Ogden CL, Johnson CL, Carroll MD, Curtin LR, Flegal KM. Prevalence of overweight and obesity among US children, adolescents, and adults, 1999-2002. Jama. 2004; 291: 2847-50.

3. Kuczmarski RJ, Flegal KM, Campbell SM, Johnson CL. Increasing prevalence of overweight among US adults. The National Health and Nutrition Examination Surveys, 1960 to 1991. Jama. 1994; 272: 205-11.

4. Subramanian S, Turner MS, Ding Y, Goodspeed L, Wang S, Buckner JH, et al. Increased levels of invariant natural killer T lymphocytes worsen metabolic abnormalities and atherosclerosis in obese mice. Journal of lipid research. 2013; 54: 2831-41.

5. Jang H, Conklin DJ, Kong M. Piecewise nonlinear mixed-effects models for modeling cardiac function and assessing treatment effects. Computer methods and programs in biomedicine. 2013; 110: 240-52.

6. Sun W, Zhang Z, Chen Q, Yin X, Fu Y, Zheng Y, et al. Magnolia extract (BL153) protection of heart from lipid accumulation caused cardiac oxidative damage, inflammation, and cell death in high-fat diet fed mice. Oxidative medicine and cellular longevity. 2014; 2014: 205849.

7. Chen Z, Watanabe RM, Stram DO, Buchanan TA, Xiang AH. High calorie intake is associated with worsening insulin resistance and beta-cell function in Hispanic women after gestational diabetes mellitus. Diabetes care. 2014; 37: 3294-300.

8. Lu Y, Qian L, Zhang Q, Chen B, Gui L, Huang D, et al. Palmitate induces apoptosis in mouse aortic endothelial cells and endothelial dysfunction in mice fed high-calorie and high-cholesterol diets. Life sciences. 2013; 92: 1165-73.

9. Kackov S, Simundic AM, Nikolac N, Celap I, Dukic L, Ruzic D, et al. The effect of high-calorie meal consumption on oxidative stress and endothelial dysfunction in healthy male adults. Physiological research / Academia Scientiarum Bohemoslovaca. 2013; 62: 643-52.

10. Kim HJ, Vaziri ND, Norris K, An WS, Quiroz Y, Rodriguez-Iturbe B. High-calorie diet with moderate protein restriction prevents cachexia and ameliorates oxidative stress, inflammation and proteinuria in experimental chronic kidney disease. Clinical and experimental nephrology. 2010; 14: 536-47.

11. Barouch LA, Gao D, Chen L, Miller KL, Xu W, Phan AC, et al. Cardiac myocyte apoptosis is associated with increased DNA damage and decreased survival in murine models of obesity. Circulation research. 2006; 98: 119-24.

12. Elmore S. Apoptosis: a review of programmed cell death. Toxicologic pathology. 2007; 35: 495-516.

13. Dhaouadi T, Sfar I, Abelmoula L, Jendoubi-Ayed S, Aouadi H, Ben Abdellah $\mathrm{T}$, et al. Role of immune system, apoptosis and angiogenesis in pathogenesis of rheumatoid arthritis and joint destruction, a systematic review. La Tunisie medicale. 2007; 85: 991-8.

14. Lee SD, Chu CH, Huang EJ, Lu MC, Liu JY, Liu CJ, et al. Roles of insulin-like growth factor II in cardiomyoblast apoptosis and in hypertensive rat heart with abdominal aorta ligation. American journal of physiology Endocrinology and metabolism. 2006; 291: E306-14.

15. Su BC, Mo FE. CCN1 enables Fas ligand-induced apoptosis in cardiomyoblast H9c2 cells by disrupting caspase inhibitor XIAP. Cellular signalling. 2014; 26: 1326-34.

16. Yang Z, Liu Y, Deng W, Dai J, Li F, Yuan Y, et al. Hesperetin attenuates mitochondria-dependent apoptosis in lipopolysaccharide-induced H9C2 cardiomyocytes. Molecular medicine reports. 2014; 9: 1941-6.

17. Lee SD, Tzang BS, Kuo WW, Lin YM, Yang AL, Chen SH, et al. Cardiac fas receptor-dependent apoptotic pathway in obese Zucker rats. Obesity. 2007; 15: 2407-15.

18. Lu MC, Tzang BS, Kuo WW, Wu FL, Chen YS, Tsai CH, et al. More activated cardiac mitochondrial-dependent apoptotic pathway in obese Zucker rats. Obesity. 2007; 15: 2634-42.

19. Hideshima T, Anderson KC. Molecular mechanisms of novel therapeutic approaches for multiple myeloma. Nature reviews Cancer. 2002; 2: 927-37. 
20. Aoyagi T, Matsui T. Phosphoinositide-3 kinase signaling in cardiac hypertrophy and heart failure. Current pharmaceutical design. 2011; 17: 1818-24.

21. Lai CH, Ho TJ, Kuo WW, Day CH, Pai PY, Chung LC, et al. Exercise training enhanced SIRT1 longevity signaling replaces the IGF1 survival pathway to attenuate aging-induced rat heart apoptosis. Age. 2014; 36: 9706.

22. Dhahri W, Drolet MC, Roussel E, Couet J, Arsenault M. Chronic high-fat diet-induced obesity decreased survival and increased hypertrophy of rats with experimental eccentric hypertrophy from chronic aortic regurgitation. BMC cardiovascular disorders. 2014; 14: 123

23. Tang RB, Chang JK, Chen HL. Can probiotics be used to treat allergic diseases? Journal of the Chinese Medical Association : JCMA. 2015.

24. Mahajan PG, Tripathi AS, Chandewar AV, Apte KG, Mazumder PM, Mahajan GD. Development of health supplement probiotic in relation to physical strength and biological activity in experimental animal. Drug research. 2014; 64: 79-84.

25. Issa I, Moucari R. Probiotics for antibiotic-associated diarrhea: Do we have a verdict? World journal of gastroenterology : WJG. 2014; 20: 17788-95.

26. Sarkar D, Ankolekar C, Pinto M, Shetty K. Dietary functional benefits of Bartlett and Starkrimson pears for potential management of hyperglycemia, hypertension and ulcer bacteria Helicobacter pylori while supporting beneficial probiotic bacterial response. Food research international. 2015; 69: $80-90$.

27. Jood S, Khetarpaul N, Goyal R. Efficacy of barley based probiotic food mixture in treatment of pathogenic E.coli induced diarrhoea in mice. Journal of food science and technology. 2012; 49: 200-6.

28. Isolauri E, Salminen S. The impact of early gut microbiota modulation on the risk of child disease: alert to accuracy in probiotic studies. Beneficial microbes. 2015; 6: 167-71.

29. Stadlbauer V. Immunosuppression and probiotics: are they effective and safe? Beneficial microbes. 2015: 1-6.

30. Ziehm D, Dreesman J, Campe A, Kreienbrock L, Pulz M. Risk factors associated with sporadic salmonellosis in adults: a case-control study. Epidemiology and infection. 2013; 141: 284-92.

31. Lin CH, Lin CC, Shibu MA, Liu CS, Kuo CH, Tsai FJ, et al. Oral Lactobacillus reuteri GMN-32 treatment reduces blood glucose concentrations and promotes cardiac function in rats with streptozotocin-induced diabetes mellitus. The British journal of nutrition. 2014; 111: 598-605.

32. Wei S-H, Chen Y-P, Chen M-J. Selecting probiotics with the abilities of enhancing GLP-1 to mitigate the progression of type 1 diabetes in vitro and in vivo. Journal of Functional Foods. 2015; 18(Part A): 473-86.

33. Panwar H, Rashmi HM, Batish VK, Grover S. Probiotics as potential biotherapeutics in the management of type 2 diabetes - prospects and perspectives. Diabetes/metabolism research and reviews. 2013; 29: 103-12.

34. Al-Salami H, Butt G, Tucker I, Skrbic R, Golocorbin-Kon S, Mikov M. Probiotic Pre-treatment Reduces Gliclazide Permeation (ex vivo) in Healthy Rats but Increases It in Diabetic Rats to the Level Seen in Untreated Healthy Rats. Archives of drug information. 2008; 1: 35-41.

35. Huang WC, Chen YM, Kan NW, Ho CS, Wei L, Chan CH, et al. Hypolipidemic effects and safety of Lactobacillus reuteri 263 in a hamster model of hyperlipidemia. Nutrients. 2015; 7: 3767-82.

36. Hsieh FC, Lee CL, Chai CY, Chen WT, Lu YC, Wu CS. Oral administration of Lactobacillus reuteri GMNL-263 improves insulin resistance and ameliorates hepatic steatosis in high fructose-fed rats. Nutrition \& metabolism. 2013; 10: 35.

37. Lu YC, Yin LT, Chang WT, Huang JS. Effect of Lactobacillus reuteri GMNL-263 treatment on renal fibrosis in diabetic rats. Journal of bioscience and bioengineering. 2010; 110: 709-15.

38. Aslam F, Bandeali SJ, Crowson C, Alam M. Cardiac Function and Diastolic Dysfunction in Behcet's Disease: A Systematic Review and Meta-Analysis. International journal of rheumatology. 2016; 2016: 9837184.

39. Kanwar M, Walter C, Clarke M, Patarroyo-Aponte M. Targeting heart failure with preserved ejection fraction: current status and future prospects. Vascular health and risk management. 2016; 12: 129-41.

40. Akiyama K, Arisawa S, Ide M, Iwaya M, Naito Y. Intraoperative cardiac assessment with transesophageal echocardiography for decision-making in cardiac anesthesia. General thoracic and cardiovascular surgery. 2013; 61: 320-9.

41. Pacileo G, Di Salvo G, Limongelli G, Miele T, Calabro R. Echocardiography in congenital heart disease: usefulness, limits and new techniques. Journal of cardiovascular medicine. 2007; 8: 17-22.

42. Khalesi S, Sun J, Buys N, Jayasinghe R. Effect of probiotics on blood pressure: a systematic review and meta-analysis of randomized, controlled trials. Hypertension. 2014; 64: 897-903.

43. de Vrese M, Schrezenmeir J. Probiotics, prebiotics, and synbiotics. Advances in biochemical engineering/biotechnology. 2008; 111: 1-66.

44. Guo Z, Liu XM, Zhang QX, Shen Z, Tian FW, Zhang H, et al. Influence of consumption of probiotics on the plasma lipid profile: a meta-analysis of randomised controlled trials. Nutrition, metabolism, and cardiovascular diseases : NMCD. 2011; 21: 844-50.

45. Yakovlieva M, Tacheva T, Mihaylova S, Tropcheva R, Trifonova K, Tolesmall $\mathrm{ka} C A$, et al. Influence of Lactobacillus brevis 15 and Lactobacillus plantarum 13 on blood glucose and body weight in rats after high-fructose diet. Beneficial microbes. 2015; 6: 505-12.
46. Oxman T, Shapira M, Klein R, Avazov N, Rabinowitz B. Oral administration of Lactobacillus induces cardioprotection. Journal of alternative and complementary medicine. 2001; 7: 345-54.

47. Huang CY, Yang AL, Lin YM, Wu FN, Lin JA, Chan YS, et al. Anti-apoptotic and pro-survival effects of exercise training on hypertensive hearts. Journal of applied physiology. 2012; 112: 883-91.

48. Barr LA, Shimizu Y, Lambert JP, Nicholson CK, Calvert JW. Hydrogen sulfide attenuates high fat diet-induced cardiac dysfunction via the suppression of endoplasmic reticulum stress. Nitric oxide : biology and chemistry / official journal of the Nitric Oxide Society. 2015.

49. Kuwabara $\mathrm{Y}$, Horie $\mathrm{T}$, Baba $\mathrm{O}$, Watanabe $\mathrm{S}$, Nishiga $\mathrm{M}$, Usami $\mathrm{S}$, et al MicroRNA-451 Exacerbates Lipotoxicity in Cardiac Myocytes and High-Fat Diet-Induced Cardiac Hypertrophy in Mice Through Suppression of the LKB1/AMPK Pathway. Circulation research. 2015; 116: 279-88.

50. Zhang L, Li N, Caicedo R, Neu J. Alive and dead Lactobacillus rhamnosus GG decrease tumor necrosis factor-alpha-induced interleukin-8 production in Caco- 2 cells. The Journal of nutrition. 2005; 135: 1752-6.

51. Adams CA. The probiotic paradox: live and dead cells are biological response modifiers. Nutrition research reviews. 2010; 23: 37-46.

52. Barbosa-da-Silva S, Fraulob-Aquino JC, Lopes JR, Mandarim-de-Lacerda CA, Aguila MB. Weight cycling enhances adipose tissue inflammatory responses in male mice. PloS one. 2012; 7: e39837.

53. He KL, Burkhoff D, Leng WX, Liang ZR, Fan L, Wang J, et al. Comparison of ventricular structure and function in Chinese patients with heart failure and ejection fractions $>55 \%$ versus $40 \%$ to $55 \%$ versus $<40 \%$. The American journal of cardiology. 2009; 103: 845-51

54. Madamanchi C, Alhosaini H, Sumida A, Runge MS. Obesity and natriuretic peptides, BNP and NT-proBNP: mechanisms and diagnostic implications for heart failure. International journal of cardiology. 2014; 176: 611-7.

55. Kuo WW, Chung LC, Liu CT, Wu SP, Kuo CH, Tsai FJ, et al. Effects of insulin replacement on cardiac apoptotic and survival pathways in streptozotocin-induced diabetic rats. Cell biochemistry and function. 2009; 27: 479-87.

56. Wong ND, Moran AE. The U.S. Prevention of Cardiovascular Disease Guidelines and Implications for Implementation in LMIC. Global heart. 2014; 9: $445-55$.

57. Lee SD, Shyu WC, Cheng IS, Kuo CH, Chan YS, Lin YM, et al. Effects of exercise training on cardiac apoptosis in obese rats. Nutrition, metabolism, and cardiovascular diseases : NMCD. 2013; 23: 566-73.

58. Lee SD, Kuo WW, Ho YJ, Lin AC, Tsai CH, Wang HF, et al. Cardiac Fas-dependent and mitochondria-dependent apoptosis in ovariectomized rats. Maturitas. 2008; 61: 268-77. 